\title{
OSSERVAZIONI AD UNA NOTA DEL PROF. STRAZZERI ${ }^{\mathrm{r}}$ ).
}

\author{
Nota di R. Occhipinti (Palermo).
}

Adunanza del 14 marzo 1926.

La Nota del prof. Strazzeri su "Le normalie e la rappresentazione delle linee di una superficie!" ${ }^{2}$ ) al $\mathrm{n} .4 \mathrm{mi}$ ha richiamato alla memoria tre mie note pubblicate tredici anni fa nel "Periodico di Matematica» [Livorno, anno XXVIII, fasc. II e V (Ottobre 1912 e Maggio I913) ed anno XXX, fasc. II (Gennaio 1915)].

In dette note mi occupo della misura della curvatura di una linea mediante il rapporto fra l'angolo di due normali principali consecutive e l'arco compreso, che ho creduto chiamare terza curvatura della linea, ne ho dato l'espressione in funzione della prima e seconda curvatura, senza ricorrere alle formole di FRENET, e ne ho fatto applicazione alle geodetiche di una superficie, enunciando cosi una proprietà comune ai doppi sistemi di linee assintotiche e caratteristiche, cioè che per queste linee la terza eurvatura geodetica $\dot{e}$ uguale, in valore assoluto, alla radice quadrata del valore assoluto della curvatura della superficie in ciascun punto.

Ho poi dato l'espressione della terza curvatura geodetica, lungo una direzione, in funzione delle curvature principali e dell'angolo di quella direzione con una linea di curvatura [formola (12) della Nota dello Strazzeri] del tutto analoga a quella di Euler per le curvature normali, ho costruito, come per queste curvature, l'ellisse indicatrice delle terze curvature geodetiche, come leggo anche nel lavoro dello Strazzeri, ed ho trovato, fra l'altro, la relazione semplicissima che lega le terze curvature geodetiche lungo due direzioni coniugate nel senso di Dupin, cioè che è costante il prodotto delle terze curvature geodetiche lungo tali direzioni, ed eguale alla curvatura della superficie.

Palermo, 23 febbraio 1926.

\section{ROBERTO OCCHIPINTI.}

I) La Redazione rammenta la disposizione per la quale riguardo alle Note di carattere polemico è ammessa la pubblicazione di una sola Nota da parte di ciascuno dei contendenti [cfr. l'avvertimento a pag. 232 del tomo XXXVI ( $2^{\circ}$ semestre 19l3) dei Rendiconti].

2) Rendiconti del Circolo Matematico di Palermo, t. 49 (I925), pp. 277-291. 